\title{
EFFECT OF ROBOTIC GAIT TRAINING VERSUS SENSORY-MOTOR SYSTEMS IN REHABILITATION OF GAIT AND BALANCE IMPAIRMENT AND FATIGUE IN MULTIPLE SCLEROSIS
}

\author{
R. Saggini ${ }^{1}$, G. Barassi ${ }^{2}$, E. Ancona ${ }^{3}$, S.M. Carmignano $^{2}$, A. Sablone $^{4}$, R.G. Bellomo $^{5}$
}

\begin{abstract}
Multiple Sclerosis (MS) is a chronic immune-mediated disease of the central nervous system, most often diagnosed in young and middle-aged subjects (two-third of which are women). Walking disturbances and fatigue are key symptoms in patients with MS, and major causes of discomfort, even in patients with mild disability since the early stages of the disease. Controversy exists about the association between fatigue and physical disability, between elevated fatigue, impaired balance and a higher risk of falls, between perceived fatigue and gait performance.

We enrolled 16 patients with relapsing-remitting MS at early stage and low or mild disability, 11 females and 5 males, aged 27.1 (range 23-34 years), randomly divided into two groups: patients in group A underwent a robotic gait rehabilitation treatment which involved the use of SPAD ${ }^{\circledR}$ (Sistema Posturale Antigravitario Dinamico, Dynamic Antigravity Postural System), patients in group B underwent a cycle of sensory-motor physical activity in our laboratory of performance enhancement; patients in both groups were subjected to neuromuscular manual therapy. All treatment were provided with 3 sessions per week for 6 weeks (for a total of 18 sessions). Patients were evaluated by administration of the Functional Independence Measure $\left(\mathrm{FIM}^{\circledR}\right)$, Expanded Disability Status Scale (EDSS), the Fatigue Severity Scale (FSS) and the Modified Fatigue Impact Scale (MFIS), and gait analysis with MTX7 ${ }^{\circledR}$ (Diagnostic Support, Rome, Italy).

Results show statistically significant improvement of the $\mathrm{FIM}^{\circledR}$ average score in all patients, reduction of the EDSS average score in all patients (but in a statistically significant manner only in group A), reduction in average scores obtained in both evaluation questionnaires of fatigue (non-significant improvement of the FSS average score in the overall sample and in both groups, statistically significant reduction of the MFIS average scores), improvement in temporal (but not spatial) gait parameters.

So body weight supported gait training is feasible and could be safely used as additional therapeutic option in MS patients with mild walking disability.
\end{abstract}

\footnotetext{
${ }^{1}$ Full Professor of Physical and Rehabilitation Medicine, Department of Medical, Oral and Biotechnologic Sciences,

“Gabriele d'Annunzio” University, Chieti-Pescara, Italy

${ }^{2}$ Chair of Physical and Rehabilitation Medicine, "Gabriele d'Annunzio” University of Chieti-Pescara, Italy

${ }^{3}$ School of Specialty in Physical and Rehabilitation Medicine, "Gabriele d'Annunzio" University of Chieti-Pescara, Italy

4 Section of Physical and Rehabilitation Medicine, "Gabriele d'Annunzio" University of Chieti-Pescara, Italy

5 Associated Professor of Physical and Rehabilitation Medicine, Department of Medicine and Science of Aging, "Gabriele

d'Annunzio" University of Chieti-Pescara, Italy
} 